\title{
A RESIDUAL PROPERTY OF CERTAIN GROUPS
}

\author{
P. F. STEBE
}

ABstract. An element $a$ of a group $G$ is called conjugacy distinguished or c.d. in $G$ if and only if given any element $b$ of $G$ either $a$ is conjugate to $b$ or there is a homomorphism $\xi$ from $G$ onto a finite group $H$ such that $\xi(a)$ and $\xi(b)$ are not conjugate in $H$. Following A. Mostowski, a group $G$ is called conjugacy separable or c.s. if every element of $G$ is c.d. A. Mostowski remarks that a direct product of c.s. groups is c.s. and proves that the conjugacy problem can be solved for finitely presented c.s. groups. N. Blackburn proves that finitely generated nilpotent groups are c.s. In this paper it is proven that a free product of c.s. groups is c.s., a free group is c.s. and that every element of infinite order in a finite extension of a free group is c.d.

An element $a$ of a group $G$ is called conjugacy distinguished or c.d. in $G$ if and only if given any element $b$ of $G$ either $a$ is conjugate to $b$ or there is a homomorphism $\xi$ from $G$ onto a finite group $H$ such that $\xi(a)$ and $\xi(b)$ are not conjugate in $H$. Following A. Mostowski [6], a group $G$ is called conjugacy separable or c.s. if every element of $G$ is c.d. A. Mostowski remarks that a direct product of c.s. groups is c.s. and proves that the conjugacy problem can be solved for finitely presented c.s. groups. N. Blackburn [1] proves that finitely generated nilpotent groups are c.s. We shall prove that a free product of c.s. groups is c.s., a free group is c.s. and that every element of infinite order in a finite extension of a free group is c.d.

I am indebted to D. S. Passman for his valuable contributions.

A few lemmas will be needed for the proofs of the theorems.

Lemma 1. Let $H$ be a subgroup of finite index in a group $G$. If $a$ is an element of $H$ and $a$ is c.d. in $H$, then a is c.d. in G.

Proof. Let $c_{i}, i=1, \cdots, m$ be a complete set of left coset representatives of $G$ modulo $H$. Let $b$ be an arbitrary element of $G$ and let $a$ be a c.d. element of $H$. Let $p_{1}, \cdots, p_{k}$ be the elements of $H$ of the form $b^{c_{i}}$. Suppose $b$ is not conjugate to $a$ in $G$. No $p_{i}$ can be conjugate to $a$ in $H$, so that there is a homomorphism from $H$ onto a finite group such that the images of $a$ and $p_{i}$ are not conjugate in the image of $H$. Let $N_{i}$ be the kernel of this homomorphism. Let $M$ be the intersection of all $N_{i}$. Since there are but finitely many $N_{i}, M$ is of finite

Received by the editors January 7, 1970.

AMS 1969 subject classifications. Primary 2048, 2052.

Key words and phrases. Group, c. s. group, residual property, conjugacy problem. 
index in $H$ and hence in $G$. Let $N$ be the intersection of all the distinct conjugates of $M$ in $G$. $N$ is normal and of finite index in $G$. Suppose there is an element $y$ of $G$ such that $a^{-1} y^{-1} b y=a^{-1} b^{y}$ is an element of $N$. There is a subscript $i$ so that $y=c_{i} x$ for some element $x$ of $H$, so that $a^{-1} x^{-1} b^{c_{i}} x$ is in $N$. This equation is impossible for $b^{c_{i}}$ not in $H$, for $H$ contains $a, x$ and $N$. Thus $b^{c_{i}}=p_{j}$ is in $H$. But $N_{j}$ contains $N$, so that $a^{-1} b^{y}$ is not in $N$ for all $y$ in $G$. Let $\xi$ be the natural mapping from $G$ onto the finite group $G / N$. Clearly, $\xi(a)$ and $\xi(b)$ are not conjugate in $G / N$. Since $b$ is an arbitrary nonconjugate of $a, a$ is c.d. in $G$.

LEMMA 2. If $g_{1}$ is a primitive element of a free group $F, g_{1}$ is c.d. in $F$.

Proof. Let $g_{1}$ be a primitive element of $F$. Let $g_{2}$ be an arbitrary element of $F$. If $g_{2}$ is conjugate to $g_{1}$, there is nothing to prove. Assume $g_{1}$ is not conjugate to $g_{2}$. Let $x_{1}=g_{1}, x_{2}, \ldots$ be a set of free generators of $F$ including $g_{1}$. If $g_{2}$ does not contain $x_{1}$, the factor group $\left(x_{1}, \cdots, ; x_{1}^{2}=x_{2}=\cdots=1\right)$ is such that the images of $g_{1}$ and $g_{2}$ are not conjugate. If $g_{2}$ contains only $x_{1}$, say $g_{2}=x_{1}^{m}$, we have $m \neq 1$ since $g_{1}$ is not conjugate to $g_{2}$. In the factor group $\left(x_{1}, \cdots\right.$, ; $x^{|m|+2}=x_{2}=\cdots=1$ ), the image of $g_{1}$ is not conjugate to the image of $g_{2}$. Thus we can assume that $g_{2}$ contains $x_{1}$ and at least one other generator of $F$. The argument to follow is a modification of the proof that free groups are residually finite in A. Kuroš [4]. Now $g_{2}$ is conjugate to an element of the form $x_{1}^{m_{1}} x_{n_{2}}^{m_{2}} \cdots x_{n_{k}}^{m_{k}}$ where $m_{1} \neq 0$ and $k>1$ and we can take $g_{2}$ to be this element. Now $g_{2}$ is cyclically reduced, so we take $1 \neq n_{2}, n_{2} \neq n_{3}, \cdots, n_{k-1} \neq n_{k}, n_{k} \neq 1$. Let $s_{i}$ be zero for $i=1$, and for $i$ greater than 1 let $s_{i}$ be the sum of the absolute values of the $m_{j}$ for $j$ less than $i$. Thus $s_{k+1}$ is defined and is the sum of the absolute values of all the $m_{i}$. Following Kuroš, we associate with $x_{n_{i}}$ the following partial permutations: if $m_{i}$ is positive, $s_{i}+1 \rightarrow s_{i}+2 \rightarrow \cdots$ $\rightarrow s_{i+1}+1$; if $m_{i}$ is negative, $s_{i+1}+1 \rightarrow s_{i+1} \rightarrow \cdots \rightarrow s_{i}+1$; but we make an exception for $x_{n_{k}}$ by replacing $s_{k+1}+1$ by 1 . Here we take $n_{1}=1$. It is clear that since $g_{2}$ is a reduced word and $n_{k} \neq 1=n_{1}$ that these partial permutations can be completed to permutations of $1,2, \cdots$, $s_{k+1}$ so that each generator of $F$ corresponds to a well defined permutation. Since $F$ is free, an assignment of the generators of $F$ determines a homomorphism of $F$. The partial permutations assigned to the generators have been so chosen that the image of $g_{2}$ in the permutation group fixes the element 1 . Now let us complete the assignment of a permutation to $x_{1}=g_{1}$. Let $x_{n_{h}}, h=h_{1}=1, h_{2}, \cdots, h_{j}$ be the successive occurrences of $x_{1}$ in the word representing $g_{2}$. With $x_{1}$ associate the partial permutations $s_{h_{i}}+1 \rightarrow s_{h_{i}}+2 \rightarrow \cdots \rightarrow s_{h_{i+1}} \rightarrow s_{h_{i}}+1$ if $m_{h_{i}}$ is positive, and $s_{h_{i+1}} \rightarrow \cdots \rightarrow s_{h_{i}}+2 \rightarrow s_{h_{i}}+1 \rightarrow s_{h_{i+1}}$ if $m_{h_{i}}$ is negative. 
If $i=j$, we replace $h_{j+1}$ by $k+1$. Now every element of $1,2, \cdots, s_{k+1}$ is involved in a cycle of the permutation corresponding to $x_{1}$ and no cycle contains less than two elements. Thus the image of $x_{1}$ or $g_{1}$ fixes no element and hence cannot be conjugate to the image of $g_{2}$.

LeMma 3. Let $F$ be a free group and let a be a nonidentity element of $F$. There is a subgroup $H$ of finite index in $F$ such that a is primitive in $H$.

Proof. Let $G$ be the cyclic subgroup of $F$ generated by an element $a$ of $F$. According to A. Karrass and D. Solitar [2], there is a subgroup $H$ of finite index in $F$ such that $G$ is a free factor of $H$, and hence $a$ is primitive in $H$.

\section{THEOREM 1. All free groups are c.s.}

Proof. Let $a$ be a nonidentity element of a free group $F$. Let $H$ be a subgroup of finite index in $F$ such that $a$ is primitive in $H$. $H$ exists by Lemma 3. By Lemma $2, a$ is c.d. in $H$. By Lemma $1, a$ is c.d. in $F$.

Let $a$ be the identity element of $F$. An element $b$ of $F$ is not conjugate to $a$ if and only if $b$ is not the identity. Since every $b \neq 1$ is c.d. in $F$ by the above, there is a homomorphism $\xi$ of $F$ onto a finite group such that $\xi(b)$ is not conjugate to $\xi(a)$.

Now $a$ is c.d. for all $a$ in $F$ so that $F$ is c.s.

THEOREM 2. Let $F$ be a normal subgroup of finite index in a group $G$. If $F$ is free and $a$ is an element of infinite order in $G, a$ is c.d. in $G$.

Proof. Let $a$ be an element of $G$. The subgroup $H$ generated by $F$ and $a$ in $G$ is of finite index in $G$. By Lemma 1 , it is sufficient to show that $a$ is c.d. in $H$.

Let $b$ be an element of $H$ not conjugate to $a$. If $a \neq b \bmod F$, then the images of $a$ and $b$ under the natural mapping from $H$ to $H / F$ are not conjugate in the abelian group $H / F$. Let $a$ have order $n$ modulo $F$. If $a^{n}$ is not conjugate to $b^{n}$ in $H$, there is a homomorphism $\xi$ from $H$ to a finite group $U$ such that $\xi\left(a^{n}\right)$ is not conjugate to $\xi\left(b^{n}\right)$ in $U$. The existence of $\xi$ follows from the fact that $a^{n}$ is c.d. in $F$ and hence in $H$. But we have $\xi(a)$ is not conjugate to $\xi(b)$ in $U$.

We will show that $a \equiv b \bmod F$ and $a^{n}$ conjugate to $b^{n}$ implies that $a$ is conjugate to $b$. Now $a^{n}$ conjugate to $b^{n}$ implies that there is an integer $r$ and an element $x$ of $F$ such that $x^{-1} a^{-r} a^{n} a^{r} x=b^{n}$, so that $\left(a^{n}\right)^{x}=b^{n}$. If we set $a_{1}=a^{x}$ we have $a_{1}^{n}=b^{n}$ and $a_{1} \equiv a \equiv b \bmod F$. Thus there is a $y$ in $F$ such that $b=a_{1} y$, and $a_{1}^{n}=b^{n}=\left(a_{1} y\right)^{n}$. Since $a_{1} y$ commutes with $a_{1}^{n}, y$ commutes with $a^{n}$. Since $F$ is free, $y$ and $a^{n}$ generate a free cyclic subgroup of $F$. There is an element $f$ in $F$ such that $f^{k}=a_{1}^{n}, f^{m}=y$, with $f \neq 1$ and $k \neq 0$ since $a$ is of infinite order in $G$. Let $U$ 
be the subgroup of $F$ generated by $f$ and $f^{a_{1}}$. $U$ is free of rank at most 2. If $U$ is free of rank $2, a_{1}^{-1} f a_{1}$ and $f$ are free generators of $U$. This is impossible for the $k$ th powers of the generators are equal for $k \neq 0$. Thus $U$ is free of rank 1 , and is free cyclic. If an element of a free cyclic group has a $k$ th root, that root is unique. Thus $f=a_{1}^{1-} f a_{1}$ and $f$ and $a_{1}$ commute. Now $y=f^{m}$ so that $y$ and $a_{1}$ commute. Thus $a_{1}^{n}=\left(a_{1} y\right)^{n}$ implies that $y^{n}=1$. Since $y$ is in $F$ and $F$ is torsion free, $y=1$ and $b=a_{1}=a^{x}$. Thus $a$ and $b$ are conjugate in $H$.

LeMma 4. The free product of finitely many finite groups is c.s.

Proof. Let $G=* A_{i}, i=1, \cdots, n$, where the $A_{i}$ are finite groups. Let $H=\times A_{i}, i=1, \cdots, n$ be the direct product of the $A_{i}$. There is a naturally defined homomorphism from $G$ to $H$ with kernel $K$. According to a theorem of A. Kuroš [3], $K$ is free since it meets no conjugate of a free factor. Since $H$ is finite, $G$ is a finite extension of a free group. Let $a$ be an element of $G$. If $a$ is of infinite order, $a$ is c.d. in $G$ by Theorem 2. If $a$ is of finite order, it is in a conjugate of a free factor by the theorem of Kuroš referenced above. Let $b$ be an element of $G$ not conjugate to $a$. If $b$ is of infinite order, $b$ is c.d. so there is a homomorphism from $G$ onto a finite group such that the images of $a$ and $b$ are not conjugate. If $b$ is of finite order, it is in a conjugate of a free factor. Thus for $a$ and $b$ of finite order there are elements $x, y$ of $G$ and elements $a$ and $b$ of the factors of $G$ such that $a=a^{x}$ and $b=b^{y}$. Since $a$ and $b$ are not conjugate, at least one of $a, b$ is not the identity. Let $X$ be the homomorphism from $G$ onto the factor containing a nonidentity $a$ or $b$ such that the kernel of $X$ is the normal closure of the other factors. If $X(a)$ and $X(b)$ are conjugate, $a$ and $b$ are in the same factor of $G$ and are conjugate in $G$, so that $a$ and $b$ are conjugate. Thus $X(a)$ and $X(b)$ are not conjugate so that $X(a)$ and $X(b)$ are not conjugate. Thus if $a$ is of finite order, $a$ is c.d. in $G$. Since every $a$ in $G$ is c.d., $G$ is c.s.

Lemma 5. Let $G$ be the free product of residually finite groups $A$ and $B$. If $g$ is an element of $G$ and $g$ is not conjugate to an element of $A$ or an element of $B, g$ is c.d. in $G$.

Proof. Let the notation be as in the statement of the lemma. Let $g_{1}$ be a cyclically reduced element conjugate to $g$. Since $g$ is not conjugate to an element of a factor, $g_{1}$ has syllable length greater than 1 . Let $h$ be any element of $G$ not conjugate to $g$. Let $g_{2}$ be a cyclically reduced element conjugate to $h$. Let $g_{i}=a_{1, i} b_{1, i} \cdots a_{n_{i}, i} b_{n_{i}, i}$, where $a_{i, j}$ is in $A, b_{i, j}$ is in $B$, adjacent nonidentity syllables are from different factors and the first and the last nonidentity syllables are 
from different factors. Set $a_{0, i}=b_{0, i}=1$. Let $N_{i, j}$ be a normal subgroup of $A$ defined as follows: $N_{i, j}=A$ if $a_{i, 1}=a_{j, 2}$ but if $a_{i, 1} \neq a_{j, 2}$ let $N_{i, j}$ be a normal subgroup of finite index in $A$ such that $a_{i, 1} \neq a_{j, 2}$ $\bmod N_{i, j}$. The $N_{i, j}$ exist since $A$ is residually finite. Let $N$ be the intersection of all the $N_{i, j}$. Since $N$ is the intersection of finitely many normal subgroups of finite index in $A, N$ is normal and of finite index in $A$. Let $M$ be the analogously defined subgroup of $B$, and let $X$ be the naturally defined homomorphism from $G$ to $H=A / N * B / M$. Clearly $X\left(g_{i}\right)$ is cyclically reduced in $H$ and $X\left(g_{i}\right)$ has the same syllable length as $g_{i}$. Suppose $X\left(g_{1}\right)$ is conjugate to $X\left(g_{2}\right)$. There is a cyclic permutation of the syllables of $X\left(g_{1}\right)$ which takes it to $X\left(g_{2}\right)$. The same cyclic permutation applied to $g_{1}$ would take $g_{1}$ to $g_{2}$. But this is impossible, for $a$ and $b$ and hence $g_{1}$ and $g_{2}$ are not conjugate in $G$. Thus $X\left(g_{1}\right)$ and $X\left(g_{2}\right)$ are not conjugate in $H$, so that $X(a)$ and $X(b)$ are not conjugate in $H$. Now $H$ is c.s. by Lemma 4 , so there is a homomorphism $Y$ of $H$ onto a finite group such that $Y X(a)$ is not conjugate to $Y X(b)$. Thus $a$ is c.d. in $G$.

THEOREM 3. The free product of two c.s. groups is c.s.

Proof. Let $a$ be an element of $G=A * B$, where $A$ and $B$ are c.s. Let $g$ be a cyclically reduced conjugate of $a$. If $g$ has syllable length greater than 1, $a$ is c.d. by Lemma 5 . Thus assume that $g$ has syllable length 1 , and thus is in a factor. Let $b$ be an element of $G$ not conjugate to $a$ and let $h$ be a cyclically reduced conjugate of $b$. If $h$ has syllable length greater than 1 , then $b$ is c.d. by Lemma 5 and there is a homomorphism from $G$ onto a finite group such that the images of $a$ and $b$ are not conjugate in the image group. Thus we may assume that $h$ has syllable length 1 and so is in a factor. Let $X$ be a homomorphism from $G$ onto a factor of $G$ containing at least one nonidentity element of $g, h$ such that the kernel of $X$ is the normal closure of the other factor. If $X(g)$ and $X(h)$ are conjugate, $g$ and $h$ are from the same factor and are conjugate in $G$. Thus $X(g)$ and $X(h)$ are not conjugate so that $X(a)$ and $X(b)$ are not conjugate. Since the factors of $G$ are c.s. groups, there is a homomorphism $Y$ from $X(G)$ to a finite group such that the images $Y X(a)$ and $Y X(b)$ are not conjugate in the finite group. Thus $a$ is c.d. Since every element of $G$ is c.d., $G$ is c.s.

A general reference for theorems not explicitly referenced is Magnus, Karrass, Solitar [5].

\section{REFERENCES}

1. N. Blackburn, Conjugacy in nilpotent groups, Proc. Amer. Math. Soc. 16 (1965), 143-148. MR 30 \#3140. 
2. A. Karrass and D. Solitar, On finitely generated subgroups of a free group, Proc. Amer. Math. Soc. 22 (1969), 209-213.

3. A. Kuroš, Die Untergruppen der freien Produkte von beliebigen Gruppen, Math. Ann. 109 (1934), 647-660.

4. - The theory of groups, GITTL, Moscow, 1953; English transl., Chelsea, New York, 1955. MR 15, 501; MR 17, 124.

5. W. Magnus, A. Karrass and D. Solitar, Combinatorial group theory: Presentations of groups in terms of generators and relations, Pure and Appl. Math., vol. 13, Interscience, New York, 1966. MR 34 \#7617.

6. A. Mostowski, On the decidability of some problems in special classes of groups, Fund. Math. 59 (1966), 123-135. MR 37 \#292.

Institute for Defense Analyses, Princeton, New Jersey 08540 